\title{
Star-Shaped Sets in Normed Spaces
}

\author{
V. Boltyanski, ${ }^{1}$ H. Martini, ${ }^{2}$ and P. S. Soltan ${ }^{3}$ \\ ${ }^{1}$ Vavilov str. 81, kv. 29 , \\ 117335 Moscow, Russia \\ ${ }^{2}$ Fakultät für Mathematik, TU Chemnitz-Zwickau, \\ Postfach 964, D-09009 Chemnitz, Germany \\ ${ }^{3}$ str. Decebal 89, app. 239, \\ Kishinev, 277032, Republica Moldova
}

\begin{abstract}
We prove a generalization of the Krasnosel'ski theorem on star-shaped sets. Using $d$-segments in $n$-dimensional Minkowski spaces instead of usual segments, the notions " $d$-visibility" and " $d$-star-shapedness" are introduced. Our main aim is to give necessary and sufficient conditions for $d$-star-shapedness in finite-dimensional normed spaces.
\end{abstract}

\section{Introduction}

Krasnosel'ski [7] proved a theorem on star-shaped sets. We recall its statement in a generalized form, as it is contained, e.g., in the book by Leichtweiss [8]:

Let $M \subset \mathbf{R}^{n}$ be a compact set. We say that $M$ is star-shaped if a point $x_{0} \in M$ exists such that for every point $x \in M$ the segment $\left[x_{0}, x\right]$ is contained in $M$. In other words, every point $x \in M$ is visible in $M$ from the point $x_{0}$. Krasnosel'ski's theorem affirms that, if every $n+1$ points of $M$ are visible in $M$ from some point, then all the points of $M$ are visible from some point of $M$, i.e., $M$ is star-shaped.

The proof of this theorem, offered by Krasnosel'ski for closed regions in $\mathbf{R}^{2}$ which are bounded by a finite number of segments, was given in a simple and general form in [3], see also [8]. This proof is based on the classical Helly theorem. For further background, we refer to the survey articles [3] and [4].

In the present paper we prove a theorem (and several consequences thereof) which generalizes Krasnosel'ski's assertion. Namely, we consider $d$-star-shapedness in a 
Minkowski space $\mathfrak{R}^{n}$, where $d$-star-shaped sets are obtained when we replace the usual segments by $d$-segments. Thus, we first recall briefly several notions connected with $d$-convexity in a Minkowski space $\mathfrak{A}^{n}$, see also [2].

\section{Basic Notions}

Let $\mathfrak{R}^{n}$ denote a (finite-dimensional) normed space or Minkowski space, whose unit ball is a compact, convex body symmetric about the origin and defining the distance between two points $x, y \in \mathfrak{R}^{n}$ in the usual way. We denote this distance by $d(x, y)=\|x-y\|$. For every two points $a, b \in \mathfrak{R}^{n}$ we denote by $[a, b]_{d}$ the $d$-segment with the endpoints $a, b$, i.e., the set of all points $x \in \Re^{n}$ satisfying the condition

$$
\|a-x\|+\|x-b\|=\|a-b\| .
$$

A set $M \subset \mathfrak{A}^{n}$ is said to be $d$-convex if for every $a, b \in M$ the inclusion $[a, b]_{d} \subset M$ holds. It is obvious that every $d$-convex set is linearly convex (i.e., it is convex in the usual sense, considering linear segments instead of $d$-segments). Since, evidently, the intersection of any family of $d$-convex sets is also $d$-convex, it is possible to define the $d$-convex hull conv ${ }_{d} X$ for every set $X \subset \mathfrak{A}^{n}$ (as the intersection of all $d$-convex sets is $d$-convex.

It should be noticed that the notion of $d$-convexity was introduced by Menger [9], de Groot [5], Aleksandrov and Zalgaller [1], Soltan and Prisakaru [12], and other mathematicians. However, except for definitions, these papers contain almost no theorems on $d$-convex sets. The theory of $d$-convex sets was mainly developed by Soltan and his students, see, e.g., [10] and [11].

A set $M \subset \mathfrak{H}^{n}$ is said to be $d$-star-shaped if a point $x_{0} \in M$ exists such that for every point $x \in M$ the $d$-segment $\left[x_{0}, x\right]_{d}$ is contained in $M$. (In other words, every point $x \in M$ is $d$-visible in $M$ from $x_{0}$.) In order to establish a necessary and sufficient condition for $d$-star-shapedness (which generalizes Krasnosel'ski's theorem), we introduce some auxiliary notions.

Let $a$ be a point of a set $M \subset \mathbf{R}^{n}$ (in particular, a boundary point). A ray $l$ emanating from $a \in M$ is said to have a free direction (with respect to $M$ ) if a point $b \in l$ distinct from $a$ exists, such that the interval $] a, b$ [ does not contain any points of $M$, i.e., $[a, b[\cap M=\{a\}$. Otherwise, the ray $l$ has a nonfree direction. The union of all rays emanating from $a \in M$ which have nonfree directions (with respect to $M$ ) is said to be the cone of nonfree directions at $a$ and is denoted by nof $f_{a} M$.

Now let $M$ be a set in a Minkowski space $\mathfrak{R}^{n}$. We say that $M$ possesses the property of internal-local $d$-conicity if for every point $a \in M$ the set $\operatorname{cl}\left(\operatorname{conv}\left(\operatorname{nof}_{a} M\right)\right)$ is $d$-convex. We now describe one more condition for a set $M \subset \mathfrak{R}^{n}$. We say that $M$ possesses the property of external-local $d$-conicity if, for every $a \in M$ and every ray $l$ emanating from $a$ and having a free direction, there is a positive number $\varepsilon$ such that for each positive $\mu<\lambda<\varepsilon$ the relation $[a+\lambda v, a+\mu v]_{d} \cap M=\varnothing$ holds, where $v$ is a vector directed along $l$. 
By him $\mathfrak{R}^{n}$ we denote the Helly dimension of the Minkowski space $\mathfrak{R}^{n}$, i.e., the minimal integer $h$ with the following property: For every finite family $\left\{M_{1}, \ldots, M_{s}\right\}$ of $d$-convex sets in $\Re^{n}(s>h+1)$ the relation $M_{1} \cap \cdots \cap M_{s} \neq \varnothing$ holds, if every $h+1$ of them has a nonempty intersection. Note that by Helly's theorem, him $\Re^{n} \leq n$.

\section{Results}

The following theorem is our main assertion. It is an analogue of Krasnosel'ski's theorem, and its proof uses some ideas from Krasnosel'ski's original proof, adapted for $d$-convexity.

Theorem. Let $M \subset \mathfrak{R}^{n}$ be a compact set that is internal-and external-local d-conic. The number him $\Re^{n}$ is denoted by $h$. The set $M$ is $d$-star-shaped if and only if for every $h+1$ points $x_{1}, \ldots, x_{h+1} \in M$ there is a point $y \in M$ such that all points $x_{1}, \ldots, x_{h+1}$ are $d$-visible in $M$ from $y$, i.e., $\left[y, x_{i}\right]_{d} \subset M$ for $i=1, \ldots, h+1$.

Proof. For every point $a \in M$, we put

$$
C(a)=\operatorname{clconv}_{d}\left(M \cap \operatorname{nof}_{a} M\right)
$$

We establish that the $d$-convex set

$$
N=\bigcap_{a \in M} C(a)
$$

is nonempty, and every point $x_{0} \in N$ has the desired property, i.e., each point $x \in M$ is $d$-visible in $M$ from $x_{0}$ :

$$
\left[x, x_{0}\right]_{d} \subset M \quad \text { for each } \quad x \in M \text {. }
$$

Let $x_{1}, \ldots, x_{h+1}$ be points of $M$. Then (according to the assumption of the theorem) there is a point $y \in M$ such that

$$
\left[y, x_{i}\right]_{d} \subset M, \quad i=1, \ldots, h+1 .
$$

Hence, $y \in\left[y, x_{i}\right]_{d} \subset$ nof $_{x_{i}} M$, and $y \in M$, i.e., $y \in C\left(x_{i}\right), i=1, \ldots, h+1$. Thus, every $h+1$ of the sets $C(a), a \in M$, has a common point. Consequently, each finite family of sets $C(a)$ has a nonempty intersection.

Now let $Q \subset \mathfrak{R}^{n}$ be a Euclidean ball containing $M$ in its interior. For every $x \in$ bd $Q$ we consider the maximal closed Euclidean ball $U(z)$ centered at $z$, which contains no points of $M$ in its interior. We take a point $a \in M \cap$ bd $U(z)$ and denote by $P(a)$ the closed half-space satisfying

$$
P(a) \cap U(z)=\{a\}
$$




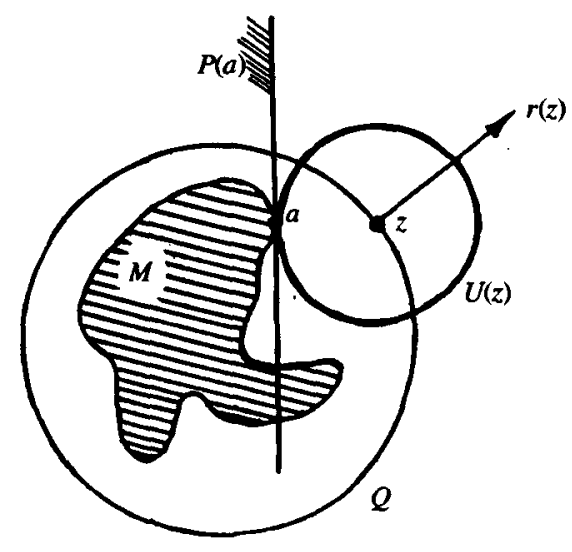

It follows from (2) that nof $M \subset P(a)$, and consequently

$$
C(a)=\operatorname{clconv}_{d}\left(M \cap \operatorname{nof}_{a} M\right) \subset \operatorname{clconv}_{d}\left(\operatorname{nof}_{a} M\right)=\operatorname{clconv}\left(\operatorname{nof}_{a} M\right) \subset P(a)
$$

(by virtue of the internal-local $d$-conicity of $M$ ). We conclude that the set $C(a)$ has no common point with the ray $r_{z}$ which emanates from $z$ and has the direction of the outward normal vector to the ball $Q$. This means that $N \cap r_{z}=\varnothing$. Since this is true for all points $z \in$ bd $Q$, the inclusion $N \subset Q$ holds. Furthermore, since $B$ is compact and $C(a)$ is closed, every finite family of sets $C(a)$ has a nonempty intersection. Thus, the whole family $C(a), a \in M$, has a nonempty intersection, i.e., $N \neq \varnothing$.

We now prove that $N \subset M$. We suppose the contrary, i.e., a point $z \in N$ not belonging to $M$ exists. Then, denoting the maximal closed Euclidean ball centered at $z$ which contains no point of $M$ in its interior by $U(z)$, we take a point $a \in M \cap$ bd $U(z)$. As above, we consider the half-space $P(a)$ satisfying (2). We conclude that nof f $_{a} \subset$ $P(a)$, and consequently relation (3) holds. This means that $z \in N \subset P(a)$, contradicting the inclusion $z \in$ int $U(z)$. This contradiction shows that $N \subset M$.

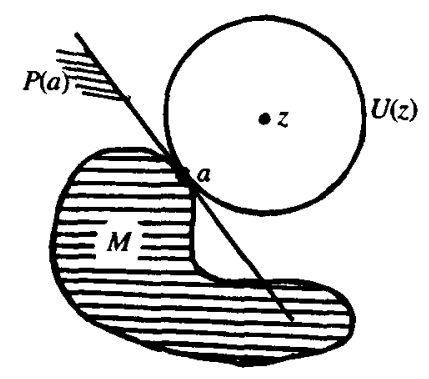

Furthermore, we establish that for all points $x_{0} \in N$ and $x \in M$ the linear segment $\left[x_{0}, x\right]$ is contained in $M$, i.e., $M$ is star-shaped in the linear sense. We suppose the contrary, i.e., there is a point $w \in\left[x_{0}, x\right]$ which does not belong to $M$. Since $M$ is compact, there is a closed Euclidean ball $B$ centered at $w$, which has no common point with $M$. Now we translate the ball $B$ in the direction $x-x_{0}$ until it has a point $a$ in 
common with $M$. We denote the translated ball by $U$. Then (int $U$ ) $\cap M=\varnothing$, and $a \in($ bd $U) \cap M$. Let $P(a)$ be the closed half-space such that $P(a) \cap U=\{a\}$ and $a \in$ bd $P(a)$. We conclude that nof ${ }_{a} M \subset P(a)$, and hence (see (3)) $C(a) \subset P(a)$, contradicting the relations $x_{0} \notin P(a), x_{0} \in N \subset P(a)$. This contradiction shows that $M$ is star-shaped in the linear sense.

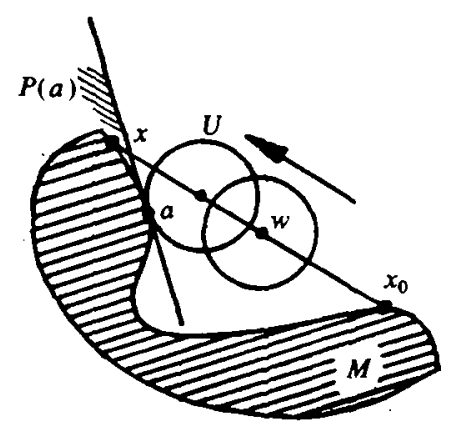

Thus, from the assumption of the internal-local $d$-conicity of $M$ we have deduced that the whole set $M$ is visible (in the linear sense) from every point $x_{0} \in N$. Now, with the help of the property of external-local $d$-conicity, we prove that $M$ is $d$-visible (from each point $x_{0} \in N$ ), i.e., for all points $x_{0} \in N, x \in M$ the $d$-segment $\left[x_{0}, x\right]_{d}$ is contained in $M$.

We assume that a point $p \in\left[x_{0}, x\right]_{d}$ exists which does not belong to $M$. Then all the points close enough to $p$ do not belong to $M$. Let $\rho$ be a positive number, such that the point $q=p+\rho\left(x_{0}-p\right)$ is not contained in $M$. We denote by $a$ that point from $[q, x]$ which is nearest to $q$ and belongs to $M$, and $v$ denotes the vector $q-a$. By virtue of the external-local $d$-conicity, an $\varepsilon>0$ exists such that $[a+\lambda v, a+\mu v]_{d} \cap M=\varnothing$ for every positive $\mu<\lambda<\varepsilon$. We notice that $\left[x_{0}, a\right] \subset M$ (according to the linear star-shapedness of $M$ which was established above).

Furthermore, since $p \in\left[x_{0}, x\right]_{d}$ and therefore $q \in\left[x_{0}, x\right]_{d}$, the following relations hold:

$$
\begin{gathered}
\left\|x-x_{0}\right\|=\|x-p\|+\left\|p-x_{0}\right\|=\|x-p\|+\|p-q\|+\left\|q-x_{0}\right\|, \\
\left\|x-x_{0}\right\|=\|x-q\|+\left\|q-x_{0}\right\| .
\end{gathered}
$$

Consequently,

$$
\|x-p\|+\|p-q\|=\|x-q\|,
$$

i.e., $p \in[q, x]_{d}$. We now denote by $k$ the number $\|q-a\| /\|q-x\|$, and by $g$ the homothety with center $q$ and ratio $k$. Then $g(x)=a$, and the point $m=g(p)$ belongs to the segment $[q, p]$. Equation (4) implies (by virtue of the relations $g(x)=a, g(p)=m$, $g(q)=q$ ) that

$$
\|a-m\|+\|m-q\|=\|a-q\| .
$$




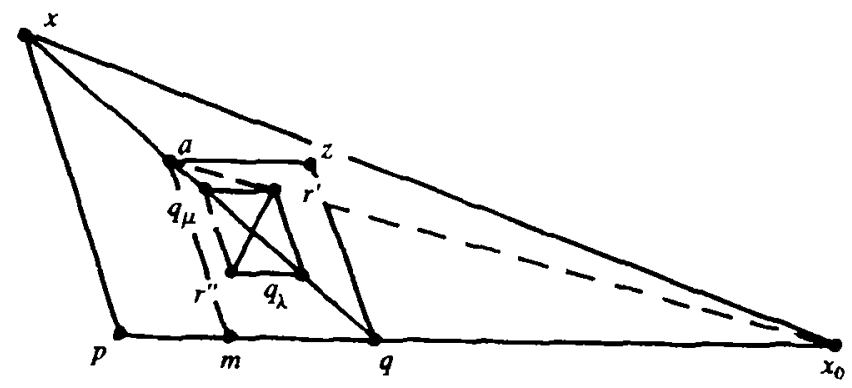

This means that $m \in[a, q]_{d}$. Furthermore, for the point $z=q+a-m$ (symmetric to $m$ with respect to the midpoint of $[q, a])$ we have

$$
\|q-z\|+\|z-a\|=\|m-a\|+\|q-m\|=\|q-a\|,
$$

i.e., $z \in[q, a]_{d}$. Furthermore, for $0<\mu<\lambda<1$ the points

$$
\begin{aligned}
& q_{\lambda}=a+\lambda(q-a)=a+\lambda v, \\
& q_{\mu}=a+\mu(q-a)=a+\mu v
\end{aligned}
$$

belong to the segment $[q, a]$. According to the property of external-local $\varepsilon$-conicity, if $\mu<\lambda<\varepsilon$, then the $d$-segment $\left[q_{\lambda}, q_{\mu}\right]_{d}$ has no common point with $M$. This $d$-segment $\left[q_{\lambda}, q_{\mu}\right]_{d}$ contains the points

$$
\begin{aligned}
& r^{\prime}=(1-\lambda) a+\mu q+(\lambda-\mu) z, \\
& r^{\prime \prime}=(1-\lambda) a+\mu q+(\lambda-\mu) m,
\end{aligned}
$$

which are (together with $q_{\lambda}, q_{\mu}$ ) the vertices of the parallelogram homothetic to conv $\{a m q z\}$ with ratio $\lambda-\mu$. Hence the segment $\left[r^{\prime}, r^{\prime \prime}\right]$ does not contain any point from $M$. However, the line passing through the points $r^{\prime}, r^{\prime \prime}$ meets the segment $\left[x_{0}, a\right]$ at the point which, for a fixed $\lambda<\varepsilon$ and $\mu \rightarrow 0$, tends to the common point of the segments $\left[x_{0}, a\right]$ and $[a+\lambda(m-a), a+\lambda(z-a)]$. Consequently, for a fixed $\lambda<\varepsilon$ and $\mu$ small enough, the segment $\left[r^{\prime}, r^{\prime \prime}\right]$ contains a point of the segment $\left[x_{0}, a\right] \subset M$ (we recall that $M$ is linearly visible from $x_{0}$, and $a \in M$ ), i.e., $\left[r^{\prime}, r^{\prime \prime}\right] \cap M \neq \varnothing$, contradicting the aforesaid. This contradiction shows that $\left[x_{0}, x\right]_{d} \subset M$.

From the theorem just established we obtain, as an immediate consequence, the classical Krasnosel'skj theorem in the formulation taken from [8].

Corollary 1. A compact set $K \subset \mathbf{R}^{n}$ is star-shaped if and only iffor every $n+1$ points $x_{1}, \ldots, x_{n+1} \in M$ there is a point $y \in M$ such that the points $x_{1}, \ldots, x_{n+1}$ are visible in $M$ from $y$, i.e., $\left[y, x_{i}\right] \subset M$ for $i=1, \ldots, n+1$.

Indeed, in Euclidean space $\mathbf{R}^{n}$ (when $d$-convexity coincides with linear convexity) the conditions of intemal-local and external-local conicity are trivially satisfied, and $\operatorname{him} \mathbf{R}^{n}=n$. 
As a second consequence, we consider the case of a compact, polyhedral region $M \subset \mathfrak{H}^{n}$, i.e., $M=\operatorname{cl}(\operatorname{int}(M)$, and the boundary of $M$ is the union of a finite number of $(n-1)$-dimensional convex polytopes which are called the facets of the region.

Corollary 2. Let $M \subset \mathfrak{R}^{n}$ be a compact, polyhedral region, each facet of which has a $d$-convex affine hull. The number him $\mathfrak{R}^{n}$ is denoted by $h$. The set $M$ is d-star-shaped if and only if each $h+1$ points of $M$ are $d$-visible in $M$ from some point of the set $M$.

Indeed, let $a \in M$. Then for a sufficiently small ball $B$, centered at $a$, the relation $M \cap B=\left(\right.$ nof $\left._{a} M\right) \cap M$ holds. Moreover, nof $_{a} M$ is a polyhedral cone (in general not convex) such that each of its $(n-1)$-dimensional faces is situated in a $d$-convex hyperplane. It follows that for every $x \in \mathfrak{R}^{n}$ the $d$-segment $[a, x]_{d}$ is either contained in $\operatorname{nof}_{a} M$, or its relative interior $\operatorname{ri}\left([a, x]_{d}\right)$ has no common point with nof $f_{a} M$. This means that the properties of internal- and external-local $d$-conicity are satisfied for $M$. It remains to apply our theorem.

Corollary 3. Let $M_{1}, \ldots, M_{s} \subset \mathfrak{A}^{n}$ (with $s>h+1$ and $h=\operatorname{him} \mathfrak{R}^{n}$ ) be sets such that:

(i) The union of every $h+1$ of them is d-star-shaped.

(ii) The set $M=M_{1} \cup \ldots \cup M_{s}$ is compact and possesses the properties of externaland internal-local d-conicity.

Then $M$ is $d$-star-shaped.

Indeed, let $x_{1}, \ldots, x_{h+1} \in M$. For every $i \in\{1, \ldots, h+1\}$ we choose an index $q_{i}$ such that $x_{i} \in M_{q_{i}}$. Then

$$
x_{1}, \ldots, x_{h+1} \in M_{q_{1}} \cup \ldots \cup M_{q_{h+1}}
$$

(if some of the indices $q_{1}, \ldots, q_{h+1}$ coincide, then we add some other sets $M_{i}$, in order to have a union of $h+1$ sets on the right-hand side of (5)). By assumption, the set $N=M_{q_{1}} \cup \ldots \cup M_{q_{h+1}}$ is $d$-star-shaped, i.e., all the points $x_{1}, \ldots, x_{h+1}$ are $d$-visible in $N$ from a point $y \in N$. Thus, every $h+1$ points of $M$ are $d$-visible in $M$ from a point $y \in M$. It remains to apply the theorem.

If, in particular, we consider the Euclidean space $\mathbf{R}^{n}$ (replacing $d$-convexity by linear convexity), then we obtain the following consequence.

Corollary 4. Let $M_{1}, \ldots, M_{s} \subset \mathbf{R}^{n}, s>n+1$. If the union of every $n+1$ of the sets $M_{1}, \ldots, M_{s}$ is star-shaped and $M_{1} \cup \cdots \cup M_{s}$ is compact, then $M_{1} \cup \cdots \cup M_{s}$ is also star-shaped.

This result was obtained by Kołodziejczyk [6]. 


\section{Examples}

Finally, we describe two examples which demonstrate that the notions of internal- and external-local $d$-conicity introduced above are independent from each other.

Example 1. In the space $\mathbf{R}^{3}$ with a fixed coordinate system $\left(x_{1}, x_{2}, x_{3}\right)$, we consider the set $\Sigma=\operatorname{conv}\left(P_{1} \cup P_{2} \cup Q\right)$, where $Q$ is described by the conditions $x_{3}=0$ and $x_{1}^{2}+x_{2}^{2}=3$, whereas $P_{1}$ and $P_{2}$ are balls of radius 1 centered at $(0,0,1)$ and $(0,0,-1)$, respectively. We denote by $\Re^{3}$ the normed space obtained from $R^{3}$ by taking $\Sigma$ as the unit ball. If now $a, b \in \mathfrak{A}^{3}$ are points such that the vector $a-b$ is parallel to the plane aff $Q$ or forms with this plane an angle not smaller than $\pi / 3$, then the $d$-segment $[a, b]_{d}$ coincides with the linear segment $[a, b]$. If this angle is smaller than $\pi / 3$, then the corresponding $d$-segment $[a, b]_{d}$ is a parallelogram. This parallelogram is situated in a plane parallel to the $x_{3}$-axis, has $[a, b]$ as its longer diagonal and, moreover, two of its sides are parallel to the plane $x_{3}=0$, and its angles are given by $\pi / 3$ and $2 \pi / 3$. It follows that the set $\Sigma$ is external-local $d$-conical. At the same time, for the point $a=(-2 / \sqrt{3}, 0,1) \in$ bd $\Sigma$ the corresponding cone $\operatorname{cl}\left(\operatorname{conv}\left(\right.\right.$ nof $\left._{a} \Sigma\right)$ ) coincides with the half-space $x_{3} \leq 3+x_{1} \sqrt{3}$. Since this half-space is not $d$-convex, the body $\Sigma$ is not internal-local $d$-conical.

Example 2. Let $\mathfrak{R}^{2}$ be the Minkowski plane in which the unit ball is the square with vertices $( \pm 1,0),(0, \pm 1)$. We denote by $Q_{0}$ the first quadrant of the plane, and by $Q_{k}$ its translate $a_{k}+Q_{0}$, where $a_{k}=\left(1 / k^{2}, 1 / k\right)$. Finally, we put

$$
P=Q_{0} \cup\left(\bigcup_{k=1}^{\infty} Q_{k}\right)
$$

and denote by $M$ the intersection of the set $P$ with the square $\left|x_{1}\right| \leq 2,\left|x_{2}\right| \leq 2$. It is easily shown that $M$ is internal-local $d$-conic, but not external-local conical.

\section{References}

1. A. D. Aleksandrov and V. A. Zalgaller, Two-Dimensional Manifolds of Bounded Curvature (in Russian). Trudy MIAN 63. M., Akademiya Nauk SSSR, Moscow, 1962.

2. V. G. Boltyanski and P. S. Soltan. Combinatorial geometry of classes of convex sets (in Russian). Uspekhi Mat. Nauk 33(1) (1978), 3-42.

3. L. Danzer, B. Grünbaum, and V. Klee, Helly's Theorem and hs Relatives. Proceeding of Symposia in Pure Mathematics, Vol. 7 (Convexity), V. Klee ed. American Mathematical Society, Providence, R1, 1963, pp. 101-180.

4. J. Eckhoff, Helly, Radon, and Caratheodory type theorems. In: Handbook of Convex Geometry, P. M. Gruber and J. M. Wills, eds. North-Holland, Amsterdam, 1993, pp. 389-448.

5. J. de Groot, Some special metrics in general topology. Colloq. Math. 6 (1958), 283-286.

6. K. Kolodziejczyk, On star-shapedness of the union of closed sets in $\mathbf{R}^{n}$. Colloq. Math. 53 (1987), 193-197.

7. M. A. Krasnosel'ski, On a criterion of star-shapedness (in Russian). Mat. Sb. 19 (1946), 309-310.

8. K. Leichtweiss, Konvexe Mengen. Springer-Verlag, Berlin, 1980. 
9. K. Menger, Untersuchungen ubber allgemeine Metrik, I, II, III. Math. Ann. 100 (1928), 75-163.

10. P. S. Soltan, The Helly theorem for $d$-convex sets (in Russian). Dokl. Akad. Nauk SSSR 205(3) (1972), 537-539.

11. P. S. Soltan, Extremal Problems on Convex Sets (in Russian). Stiinca, Kishinev, 1976.

12. P. S. Soltan and K. Prisakaru, The Steiner problem on graphs (in Russian). Dokl. Akad. Nauk. SSSR 198(I) (1971), 46-49.

Received July 20, 1994, and in revised form February 1, 1995. 